\title{
RECOGIDA DE DATOS Y MODELIZACIÓN DE LA DEMANDA DE TRANSPORTE EN LA REPÚBLICA DOMINICANA: REVISIÓN DE LITERATURA
}

\author{
Data collection and modeling of the transportation \\ demand in the Dominican Republic: Literature Review \\ Dr. ING. Lissy La Paix Puello ${ }^{1}$
}

\begin{abstract}
Cómo citar: La Paix Puello, L. (2018). Recogida de datos y modelización de la demanda de transporte en la República Dominicana: Revisión de literatura. Ciencia, Ingenierías y Aplicaciones, 1(1), 79-98. doi:http://dx.doi.org/10.22206/cyap.2018.v1i1. pp79-98
\end{abstract}

\section{Resumen}

La apertura de un nuevo modo de transporte genera cambios en la movilidad de los viajeros en su área de servicio. Sin embargo, la omisión de efectos de hábitos e inercia puede producir resultados sesgados en la predicción de la demanda. Los viajeros reaccionan diferente en cuanto a la selección de modos de transporte según sean sus hábitos de selección modal más o menos fuertes. Igualmente, la literatura ha demostrado que el comportamiento pasado es la mejor predicción del comportamiento futuro. Por tanto, el principal objetivo del presente documento es revisar la literatura correspondiente a la modelización de la demanda de viajeros entre distintos modos de transporte de la ciudad de Santo Domingo. Igualmente, se incluye una breve reseña sobre el uso de nuevas tecnologías, tales como los GPS y smartphones para la recogida de datos, y un análisis de accesibilidad. Finalmente, se describen los modelos de demanda para analizar futuras políticas de transporte urbano en la República Dominicana.

Palabras clave: modelización de demanda de transporte; inercia de pasajeros; transporte; movilidad sostenible; efectos de hábitos.

1 Assistant Professor, University of Twente. Correo electrónico: 1.c.lapaixpuello@utwente.nl 


\begin{abstract}
The use of new transportation modes generate a change in the mobility of passengers in their service area. However, omission of habit effects can result in biased results in the demand predictions. Travelers react differently about the selection of transportation modes depending on their selection mode habits. Similarly, the literature has demonstrated that past behavior is the best predictor of future behavior. For this reason, the main objective of this paper is to review the literature corresponding to travelers demand modeling among different transportation modes in Santo Domingo. Similarly, a brief summary of the use of new technologies such as GPS and smartphones for data collection, and an accessibility analysis is included. Finally, demand models to analyze future urban transportation policies in the Dominican Republic are discussed.
\end{abstract}

Keywords: transportation demand modeling; passengers inertia; transportation; sustainable movability; habit effects 


\section{Introducción}

El uso de sistemas GPS Smartphone para capturar la movilidad está teniendo un auge importante en la investigación del transporte. Se citan casos en diversos lugares, tales como Holanda, con el Dutch Mobile Mobility Panel'; Italia, con un análisis de 'returners' y 'explorers' entre ciudades; y Nairobi, donde se ha utilizado las señales de Smartphone para mejorar el transporte informal.

A través de datos GPS, cuando el diseño lo permite, un análisis de movilidad puede ser realizado a nivel micro. En tal caso, la recogida de datos consiste primeramente en el diseño de una aplicación que hace un seguimiento del movimiento del usuario. Este movimiento es capturado en coordenadas GPS como una serie de puntos que marcan la ruta seguida por el usuario durante días o semanas. El modo de transporte y el propósito pueden ser inferidos mediante un algoritmo interno de la aplicación. Posteriormente, los usuarios pueden proceder a corregir la información referida a número de viajes, modo de transporte, propósito de viaje, etc., mediante interacción web. La información es almacenada en un servidor, desde donde es descargada para realizar los análisis de movilidad. El análisis y la modelización permitiría conocer y predecir las rutas, las paradas, los destinos, los tiempos de viaje y los tiempos de espera de los usuarios. Igualmente, patrones de movilidad, en cuanto a modo y destino, también pueden ser analizados y optimizados (Bernardi et al.; Thomas et al., 2018).

Por otro lado, los datos GSM no siempre permiten el seguimiento a nivel micro (individual) de los usuarios debido a condiciones de privacidad. Sin embargo, ofrecen velocidad y densidad de la señal, haciendo posible identificar aglomeraciones en un contexto temporal y espacial; por ejemplo, eventos especiales a horas determinadas, congestión de tráfico, horas punta, etc. 
Algunos países han utilizado Twitter para 'escuchar las ciudades'. Mediante extracción de palabras clave se reconstruye información que representa satisfacción con servicios y lugares. En este sentido, el uso de big data se aplica a estimaciones de atracción y generación de viajes a determinados destinos. Igualmente, el uso de datos TomTom para analizar la accesibilidad dinámica en carretera permite conocer los tiempos de viajes a intervalos temporales mínimos; de esta manera, los usuarios de la vía pueden planificar su viaje evitando la congestión. El concepto accesibilidad se refiere a la facilidad de alcanzar destinos en determinado intervalo de tiempo, considerando que cada destino posee un peso dado por los empleos disponibles, las zonas comerciales, etc. El presente documento contiene un resumen de los tópicos principales para alcanzar una planificación del transporte basada en una modelización eficiente y sofisticada. Esta modelización de la demanda, a su vez, se apoya en datos reales recogidos con precisión.

\section{Planificación del transporte y el contexto dominicano}

La planificación del transporte constituye un rol de suma importancia en la economía de un país. La inversión promedio en infraestructura de transporte (ferrocarril y carretera) entre los países miembros de la OECD varía desde el 50\% hasta el 250\% del GDP (Producto Interior Bruto-Gross Domestic Product). Con la crisis económica, el sector transporte se encuentra en recesión. En la Unión Europea, el número de pasajeros-kilómetros en ferrocarril aumentó un $0.2 \%$ en 2010, después de un descenso del 2\% en 2009. Fuera de la Unión Europea, en Rusia descendió un 8\%, incrementó un 4\% en Ucrania y tan solo un $0.4 \%$ en China. En cuanto al transporte privado, este también se encuentra en descenso en países como Estados Unidos y Japón; por el contrario, en Alemania se encuentra en aumento.

Con el paso de los años, en distintas partes del mundo, principalmente en Europa, el transporte público ha dejado de ser visto como un 
indicador de bajos recursos económicos. La República Dominicana es la segunda economía más grande de Centroamérica y el Caribe ${ }^{3}$ y es el destino turístico más visitado del Caribe. No obstante, la situación actual del transporte en la República Dominicana se describe como un círculo vicioso, donde el aumento del transporte privado aumenta la congestión y reduce la velocidad del transporte público por carretera. El metro surge como solución a este círculo, pero se necesita un modo de transporte público masivo sostenible en tres aspectos: económico, social y medioambiental.

Según el informe Brundtland, "Nuestro Futuro Común" (1987), el desarrollo sostenible se define como "el desarrollo que satisface las necesidades del presente sin poner en peligro la capacidad de las futuras generaciones para satisfacer sus propias necesidades". Por tanto, un sistema de transporte sostenible es el que:

- Permite responder a las necesidades básicas de acceso y desarrollo de individuos, empresas y sociedades, con seguridad y de manera compatible con la salud humana y el medioambiente, y fomenta la igualdad dentro de cada generación y entre generaciones sucesivas;

- Resulta asequible, opera equitativamente y con eficacia, ofrece una elección de modos de transporte y apoya una economía competitiva, así como el desarrollo regional equilibrado;

- Limita las emisiones y los residuos dentro de la capacidad del planeta para absorberlos, usa energías renovables al ritmo de generación y utiliza energías no renovables a las tasas de desarrollo de sustitutivos de energías renovables, mientras se minimiza el impacto sobre el uso del suelo y la generación de ruidos.

A tales efectos, el transporte urbano produce impactos adversos sobre el equilibrio buscado en el desarrollo sostenible. Los principales efectos se presentan en el siguiente esquema:

3 Central Intelligence Agency, CIA. (2010). «Rank Order-GDP (purchasing power parity)» (en inglés, HTML). The World Factbook (consultado el 2 de junio de 2011). 
Figura 1. Efectos del transporte y movilidad sostenible

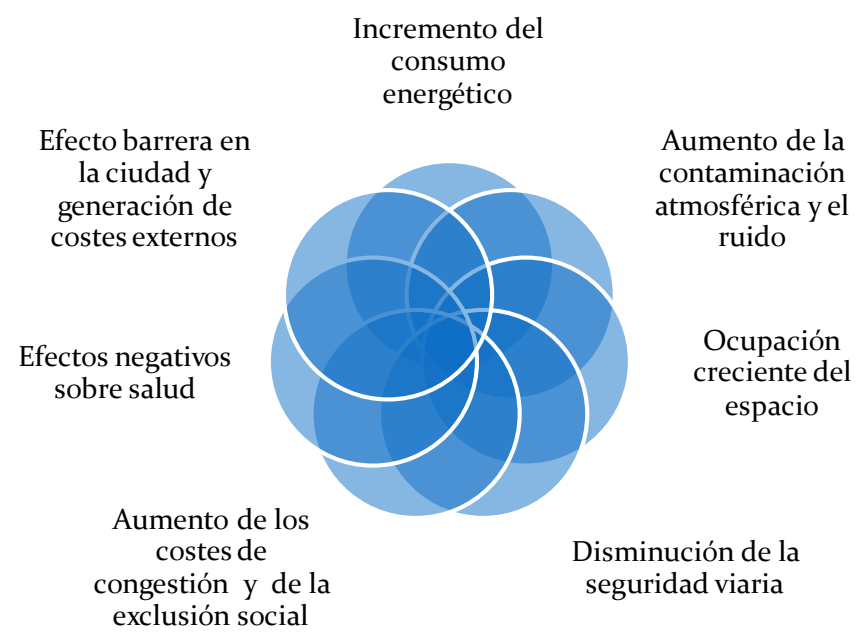

La movilidad sostenible supone el desplazamiento de las personas y los bienes según una planificación viable que permita un uso racional de los medios de transporte por los diferentes sectores de la sociedad. El fenómeno urban sprawl, también conocido como suburban sprawl, es un concepto multifacético que incluye la dispersión fuera de la ciudad y sus suburbios hacia zonas de baja densidad, con desarrollo urbano en territorio rural dependiente del automóvil y con características de diseño que promueven el uso elevado del automóvil. Como resultado, se critican ciertas desventajas, incluyendo:

- Largas distancias al trabajo

- Elevado uso del automóvil

- Inadecuadas instalaciones de salud, cultural, ocio, etc.

- Más altos costes de infraestructura por persona.

\section{Relación de la estructura urbana y la movilidad}

El proceso de expansión en muchas ciudades europeas genera cambios en la movilidad. Tanto es así que los ciudadanos utilizan su 
entorno en función de la accesibilidad al transporte de que disponen. Las 'barreras urbanas' condicionan los desplazamientos; entre estas barreras cabe mencionar la ausencia de espacios para los peatones, la no disponibilidad de redes de transporte entre municipios de la periferia y la escasa accesibilidad al transporte público con seguridad y fiabilidad en tiempo. Con el paso de los años, las ciudades van cambiando y requieren cambios en la infraestructura del transporte.

Existe un creciente interés en el estudio de la relación entre Entorno Urbano (EU) y movilidad. La relación entre la forma urbana y la movilidad es un fenómeno muy importante, debido a los muchos efectos que se observan dentro del mismo fenómeno. Muchas dimensiones de la movilidad se han estudiado, así como también muchas dimensiones de las formas urbanas.

Estos estudios han sido motivados por la posibilidad de diseñar políticas de transporte eficientes para la demanda del transporte, ajustadas a las pautas de movilidad. La asociación existente entre estos dos conjuntos (EU-Movilidad) es de naturaleza compleja, motivando a los investigadores del transporte a analizarla desde diversos puntos de vista. Por ejemplo, la forma urbana está compuesta por diversos elementos: accesibilidad al transporte público, disponibilidad de zonas peatonales y ciclistas, usos mixtos del suelo, densidad del viario, tamaño de bloques de viviendas y tipos de viviendas, entre otros. Asimismo, se han estudiado muchos factores de la movilidad, como son: disponibilidad de automóvil, frecuencia de viajes, hora, ruta, modos de transporte, propósito del viaje y el 'tour'.

Resulta difícil resumir los resultados de las investigaciones realizadas en los últimos quince años con relación al tema en cuestión. Uno de los primeros enfoques en los estudios previos ha sido la relación entre la disponibilidad de automóvil en el hogar y la densidad de suelo residencial (Pushkarev \& Zupan, 1977), donde esta última es asociada con un elevado uso del transporte público, el tamaño del hogar y 
el nivel de ingresos (Alonso, 1964). Otro estudio más reciente analizó la relación entre la disponibilidad de automóvil y la localización residencial (Howell \& Páez, 2009), el tipo de vivienda, las características sociodemográficas y la estructura del hogar. En varios estudios la relación entre la estructura urbana y los patrones de movilidad ha sido estudiada desde la dimensión de la generación de viajes; por ejemplo, el total de viajes por modo y/o motivo y las actividades desarrolladas durante un período específico (Bhat \& Koppelman, 1999). En la mayoría de los estudios se encontró que los factores socioeconómicos, individuales y del hogar afectaban significativamente la generación de viajes (Boarnet $\&$ Sarmiento, 1998) y el desarrollo de actividades de ocio (La Paix et al., 2018).

La importancia de las características de la forma urbana, en comparación con las variables socioeconómicas, cuando se analiza la distancia y la frecuencia del viaje, fue estudiada por Hanson (1982), quien encontró que estas características explican mejor la generación de viajes que las variables territoriales. En contraste, Kitamura et al. (1997) concluyeron que el número total de viajes está significativamente asociado con las variables demográficas y socioeconómicas, pero no con las variables territoriales y de forma urbana. En contraste, el uso del transporte público y los modos no motorizados, y por tanto el reparto modal, está relacionado con las características territoriales.

Un estudio reciente encontró que existe una fuerte influencia de las características del individuo y el entorno urbano sobre la elección modal y la distancia del viaje (Dieleman et al., 2002). Pocos estudios han analizado el efecto de los usos de suelo en términos de las características de la zona (Kitamura et al., 1997). Algunos autores definen la zona en términos del nivel de ingresos por habitante/año (Paez et al., 2007), distinguiendo entre zonas ricas y zonas de menor ingreso. Otros autores emplearon la tipología de barrio de acuerdo a la estructura urbana; se consideraron dos tipos: tradicional y suburbana. La zona tradicional difiere de la zona suburbana en la estructura de calles en forma de malla, 
la alta densidad residencial y la disponibilidad de comercios y servicios (White Mountain Survey, 1991; Friedman et al., 1994; Ewing, 1993; Handy et al., 2005). En cambio, Bhat \& Srinivasan (2005) analizaron cuatro tipos de variables de estructura urbana: tipo de barrio (CBD, urbano, suburbano y rural), densidad de uso mixto del suelo, proporción de viviendas aisladas y apartamentos, y distrito de residencia. De estas, solo el tipo de barrio y el distrito resultaron significativos.

En los trabajos previos se demuestra que diversos factores influencian la relación existente entre la movilidad y el entorno urbano. Estos factores dependen, por supuesto, de cuál dimensión o característica de la movilidad se considera y cómo se define el entorno urbano. A pesar de esto, en muy pocos trabajos se ha analizado el individuo como unidad de análisis de la elección "ordenada" de viajes. Un estudio reciente analizó la relación entre la expansión urbana y la movilidad al trabajo en la comunidad de Madrid (García-Palomares, 2009). No obstante, la diferencia entre este estudio y el presente trabajo empieza por la unidad de análisis: en el estudio de García-Palomares (2009) la unidad de análisis fue el municipio. Se analizaron solo los municipios suburbanos y se excluyó el municipio de Madrid, mientras que en el presente estudio se consideran tres municipios, incluyendo Madrid, y la unidad de análisis es el individuo. Segundo, en aquel estudio se utilizó una regresión múltiple para la estimación de las variables dependientes y en el presente estudio se utiliza un modelo de elección discreta, que representa las decisiones del individuo con mayor exactitud. Cabe resaltar que algunas decisiones, tales como: viajar o no, cuántos viajes realizar, con cuáles medios, por cuáles motivos, etc., son elecciones entre cantidades discretas. Por lo tanto, los modelos de elección discreta constituyen la herramienta más apropiada.

Tercero, normalmente se estudia únicamente la movilidad relativa al trabajo (García-Palomares, 2009). Sin embargo, el estudio de los viajes según motivos se encuentra más vinculado con el desarrollo urbano, ya que con los diferentes destinos se capta mejor el uso mixto 
del suelo -residencial, comercial y lugares de trabajo-. En este sentido, se requieren proyectos de investigación que analicen el impacto de la estructura urbana en la movilidad. Estos proyectos desarrollarán una metodología concerniente al análisis espacial de la accesibilidad y la modelización de la demanda de transporte.

\section{Medidas de accesibilidad al transporte público}

Se entiende como viaje el desplazamiento entre un origen y un destino con un motivo específico. En este contexto trataremos los viajes urbanos sin estadía nocturna fuera del domicilio. De acuerdo con esto, un viaje es generado por la necesidad de participar en actividades; el viaje es un medio, no es un fin en sí mismo. Junto con los desarrollos urbanos la población se expande de centros monocéntricos a policéntricos (Gordon, 1986; Small, 1992; Clark, 1994; McDonald, 1994; Cervero, 1997), generando asentamientos desprovistos de servicios de transporte adecuados. A su vez, las ciudades se encuentran dentro de una 'development trap', que conlleva a más congestión de tráfico y a mayor tiempo de viaje, debido a la adquisición de automóviles privados para trasladarse desde los nuevos asentamientos urbanos (Ortuzar \& Willumsen, 2011).

Por lo tanto, es importante asegurar un nivel adecuado de accesibilidad al transporte, entendiendo por accesibilidad la facilidad con la cual se alcanzan diferentes destinos desde el mismo punto (origen) (Geurs \& Wee, 2013; Geurs et al., 2012; Geurs \& Van Wee, 2004b). Existen diversas maneras de medir la accesibilidad. Una de las más utilizadas es 'location based measures', cuya traducción literal al español sería 'medidas de accesibilidad basadas en la localización'. Geurs \& Van Wee (2004a) definen cuatro perspectivas básicas para medir la accesibilidad: individuales, basadas en infraestructura, basadas en la localización y basadas en la utilidad. En las medidas basadas en la localización, la definición de accesibilidad entre las zonas $i$ e $j$, una persona que viaja entre $i$ e $j$ experimenta un factor de resistencia, 
dado por el coste generalizado del transporte. Este coste se encuentra afectado por los coeficientes de la función de utilidad. La función de utilidad del coste generalizado representa los elementos relacionados con la zona, como los usos de suelo y el número de actividades en destino; o con el nivel de servicio en sí mismo. Tales características de los modos de transporte, el propósito de viaje o una combinación de estas y el transporte pueden afectar igualmente la utilidad del viaje.

El concepto Transit Oriented Development (TOD) es bien conocido en Estados Unidos y parcialmente conocido en Europa (La Paix \& Geurs, 2015; La Paix \& Geurs, 2016). TOD consiste en el uso mixto de áreas residenciales o comerciales diseñadas para maximizar el acceso al transporte público y además incorporar elementos que promuevan el uso del transporte público (Cervero \& Kockelman, 1997a; Cervero et al., 2004; Cervero \& Kockelman, 1997b). Un barrio TOD usualmente tiene un centro con una estación de tren, metro, tranvía o parada de autobús, rodeado por el desarrollo de densidad relativamente alta, con menor densidad hacia afuera del centro. Los TODs generalmente se encuentran dentro de un radio de 400 a 800 metros de la estación de TP; se considera esta una escala apropiada para el peatón, ya que todos los viajes en transporte público implican una parte en la que hay que caminar.

De acuerdo con lo anterior, el área de servicio del transporte público urbano ha sido motivo de numerosas investigaciones (El-Geneidy et al., 2009; Cardozo et al., 2010; Gutiérrez et al., 2011). Delmelle \& Casas (2012) realizan un análisis espacial de la accesibilidad a las estaciones del bus rápido BRT en Cali, Colombia. Utilizan un análisis de las distancias en red, resaltando las ventajas con respecto a distancias Euclidianas. Similarmente, Gutiérrez et al. (2011) estudian la correlación especial a través del efecto 'distance decay', donde utilizan Sistemas de Información Geográfica (GIS) y modelos de regresión múltiple. Dichas investigaciones han destacado la importancia de la distancia a las estaciones de transporte público y el efecto declive 
('distance decay') de la demanda de viajeros conforme aumenta la distancia desde el domicilio hasta la estación de transporte. Por su parte, las medidas de accesibilidad combinadas con modelos de elección discreta han recibido menos atención.

\section{Modelización de la demanda de transporte}

En la modelización del transporte, la recolección de datos juega un papel primordial. La recogida de datos transversal ha sido el método tradicional de recolección de la movilidad, donde los viajeros completan un diario de viajes. No obstante, se corre el riesgo de perder mucha información relativa a decisiones repetitivas o costumbres que influencian la elección. De tal manera, las encuestas longitudinales o encuestas panel nos permiten representar mejor la correlación entre las decisiones tomadas por el mismo individuo en distintos períodos de tiempo o en el mismo lugar.

Según Cherchi (2009), conectar el comportamiento pasado es la mejor predicción del comportamiento futuro. Con este fin, los modelos de elección discreta mixtos han demostrado una alta capacidad para representar la realidad de una manera matemática. Particularmente, los modelos mixtos con efectos aleatorios confieren mayor robustez al análisis. Al mismo tiempo, mucho trabajo es aún requerido para ampliar las aplicaciones de los modelos mixtos. Por tanto, la presente investigación representará contribuciones empíricas a este campo a través del desarrollo de un modelo mixto de elección modal, incluyendo efectos temporales.

De un modo similar, Yáñez et al. (2010) muestran las ventajas significativas de los datos longitudinales en el análisis del Transantiago de Chile. El estudio analiza los efectos de inercia y shock en los viajeros durante cuatro olas de recogida de información. Otros estudios resaltan la importancia de referencias innovadoras para la planificación del transporte público en Latinoamérica; por ejemplo, el acceso 
en bicicleta a las estaciones del metro (de Souza et al., 2017). Sin embargo, los países europeos siguen siendo pioneros en encuestas de panel o longitudinales de amplia muestra (La Paix et al., 2017).

En la actualidad, los modelos de demanda desagregados constituyen la herramienta de análisis adecuada para abordar el problema de modelar la demanda por transporte. Estos se basan en el análisis del comportamiento de cada consumidor individual y cuentan con una base teórica sólida dentro del marco de la microeconomía de las elecciones discretas (McFadden, 1981) y de la teoría de la utilidad aleatoria (Domencich \& McFadden, 1975).

Según esta teoría, cada individuo $n$ tiene una función de utilidad Una asociada a cada una de las alternativas i, escogiendo aquella alternativa que maximiza su utilidad. Esta función, propia del individuo, puede dividirse en una componente sistemática $V_{\text {in }}$, que recoge el efecto de las variables explicativas (atributos medibles u observables por parte del modelador) y una componente aleatoria $\varepsilon_{\text {in }}$, que intenta recoger todos aquellos efectos no incluidos en la componente sistemática de la función de utilidad:

$$
\begin{array}{ll}
\mathrm{U}_{n i} \geq \mathrm{U}_{n j} \quad \forall \mathrm{j} \in \mathrm{C}(n), j \neq i & \text { Eq. } 1 \\
U_{n i}=U\left(X_{n i}\right) & \text { Eq. } 2 \\
U_{n i}=V_{n i}+\varepsilon_{n i} & \text { Eq. } 3
\end{array}
$$

Los modelos más utilizados en la actualidad son el Logit Multinomial (McFadden, 1974). Es en este contexto que en los últimos años aparecen los modelos Mixed Logit. La idea central de este tipo de modelos es considerar más de una componente aleatoria; de esta forma, además de una componente Gumbel iid, con lo cual el modelo básico 
es Logit, se agregan otras componentes que permiten modelar correlación y/o heteroscedasticidad. Esto permite ganar generalidad, pero la estimación deja de ser simple, como en el caso del modelo Logit y, al igual que en el caso del Probit, se requiere simulación.

La probabilidad de elegir la alternativa corresponde a la integral de la probabilidad condicional sobre todos los posibles valores de $\beta$, lo que depende de los parámetros de su distribución, esto es:

$$
P_{n j}=\int L_{n i}(\beta) f(\beta) d \beta
$$

Los modelos Mixed Logit han irrumpido con mucha fuerza en el ambiente teórico de la modelación de demanda por transporte en los últimos años. Los modelos de demanda a desarrollar para captar los cambios en el comportamiento de los usuarios deben contener efectos temporales. A través de estos modelos se puede representar el impacto de nuevas medidas de transporte, tales como la apertura de una nueva línea de transporte público o nuevos desarrollos urbanos.

\section{Conclusiones}

En la República Dominicana, la movilidad de pasajeros representa uno de los más importantes retos para la economía del país. Las políticas de transporte deberían enfocarse en el desarrollo de espacios urbanos mixtos, las zonas de trabajo y estudios, y evitar los desarrollos urbanos aislados. A su vez, se debe dotar a las áreas suburbanas de un servicio de transporte público adecuado que potencie los viajes multi-etapas desde las zonas más alejadas del centro. Y finalmente, tomando en cuenta que las actitudes y percepciones influyen en la estructura del tour, las entidades del transporte deben optimizar el proceso de implementación de las medidas transporte para asegurar el éxito de las mismas. Por ejemplo, numerosos estudios analizan la influencia del entorno urbano sobre dos dimensiones de la movilidad: 
número de viajes y tipo de tour (La Paix et al. 2010; La Paix et al. 2012; La Paix ; La Paix et al. ; Monzón et al. 2008).

El reto de la planificación del transporte es diseñar herramientas eficientes económicamente. La recogida de datos no es siempre económicamente factible. Es por esto que el uso de datos pasivos implica un gran atractivo. Enfrentamos un tiempo de popularidad en el uso de big data, redes sociales, señales GPS y GSM datos e información en la planificación del transporte. República Dominicana cuenta con estas herramientas, ¿cómo podemos utilizarlas? Recurrir a estas herramientas para mejorar el transporte público sería un competente punto de inicio.

El reto de la planificación del transporte es diseñar herramientas eficientes a nivel económico. La recogida de datos no es siempre económicamente factible. Es por esto que el uso de datos pasivos implica un gran atractivo. Enfrentamos un tiempo de popularidad en el uso de big data, redes sociales, señales GPS y GSM datos e información en la planificación del transporte. República Dominicana cuenta con estas herramientas, pero ¿cómo podemos utilizarlas? Recurrir a estas herramientas para mejorar el transporte público sería un competente punto de inicio.

\section{Referencias}

Alonso, W. (1964). Location and Land Use: toward a General Theory of Land Rent. Cambridge: Harvard University Press.

Bernardi, S., Puello, L.L.P. \& Geurs, K.T. (submitted). The evaluation of Dutch cycling patterns: evidence from smartphone data. Journal of Transport and Land Use.

Bhat, C. \& Koppelman, F. (1999). A retrospective and prospective survey of time-use research. Transportation, 26(2): 119-139.

Bhat, C., Srinivasan, S. (2005). A multidimensional mixed ordered-response model for analyzing weekend activity participation. 
Transportation Research Part B: Methodological, 39(3): 255278.

Boarnet, M., Sarmiento, S. Can land-use policy really affect travel behaviour? A study of the link between non-work travel and landuse characteristics. Urban Studies, 35(7): 1155-1169.

Cardozo, O., Gutiérrez Puebla, J. \& García Palomares, J.C. Influencia de la morfología urbana en la demanda de transporte público. Geofocus, (10)4.

Cervero, R. \& Kockelman, K. (1997a). Travel Demand and the 3 Ds: Density, Diversity and Design. Transportation Research Part D, Vol. 2, 199-219.

Cervero, R. \& Kockelman, K. (1997b). Travel demand and the 3Ds: Density, diversity, and design. Transportation Research Part D: Transport and Environment, 2(3), 199-219.

Cervero, R., Murphy, S., Ferrell, C., Goguts, N. \& Tsai, Y.H. (2004). Transit-Oriented Development in America: Experiences, Challenges and Prospects. In, vol. TCRP Report 102. Transportation Research Board, Washington D.C.: National Research Council.

Cherchi, E. Modelling individual preferences, state of the art, recent advances and future directions. In: 12th International Conference on Travel Behaviour Research (IATBR), Jaipur, India, 13-18 December, 2009.

De Souza, F., La Paix Puello, L., Brussel, M., Orrico, R. \& van Maarseveen, M. (2017). Modelling the potential for cycling in access trips to bus, train and metro in Rio de Janeiro. Transportation Research Part D: Transport and Environment, 56, 55-67.

Delmelle, E.C. \& Casas, I. Evaluating the spatial equity of bus rapid transit-based accessibility patterns in a developing country: The case of Cali, Colombia. Transport Policy, 20(0): 36-46.

Dieleman, F.M., Dijst, M. \& Burghouwt, G. Urban form and travel behaviour: micro-level household attributes and residential context. Urban Studies, 39(3): 507-527. 
Domencich, T. \& McFadden, D. (1975). Urban travel demand: a behavioral analysis / Thomas A. Domencich and Daniel McFaden. Vol. Book, Whole. Amsterdam: North-Holland, New York: American Elservier.

El-Geneidy, A.M., Tétreault, P.R. Surprenant-Legault, J. (2018) Pedestrian access to transit: Identifying redundancies and gaps using a variable service area analysis. Automated Vehicles Symposium. San Francisco, CA.

Ewing, R. (1993). Transportation service standards-as if people matter. Transportation Research Record 1400, 10-17.

Friedman, B., Gordon, S.P. \& Peers, J.B. (1994). Effect of neotraditional neighborhood design on travel characteristics. In Transportation Research Record: Journal of the Transportation Research Board, No. 1446, Transportation Research Board of the National Academies, Washington, D.C.,1466, 63-70.

García-Palomares, J.C. Urban sprawl and travel to work: the case of the metropolitan area of Madrid. Journal of Transport Geography, 18(2): 197-213.

Geurs, K., Bok, M.d. \& Zondag, B. (2012). Accessibility benefits of integrated land use and public transport policy plans in the Netherlands. In: Geurs, K.T., Krizek, K. \& Reggiani, A. (eds.) Accessibility Analysis and Transport Planning: Challenges for Europe and North America.

Geurs, K. \& Wee, B.v. (2013). Accessibility: perspectives, measures and applications. In: B. van Wee, J.A.A., D. Banister (ed). The Transport System and Transport Policy: An Introduction.

Geurs, K.T., \& Van Wee, B. (2004a). Accessibility evaluation of landuse and transport strategies: review and research directions. Journal of Transport geography, 12: 127-140.

Geurs, K.T. \& Van Wee, B. (2004b). Land-use/transport interaction models as tools for sustainability impact assessments of transport in- 
vestments: review and research directions. European Journal of Transport and Infrastructure Research, 4(3): 333-355.

Gutiérrez, J., Cardozo, O.D. \& García-Palomares, J.C. Transit ridership forecasting at station level: an approach based on distance-decay weighted regression. Journal of Transport Geography, 19(6): 1081-1092.

Handy, S., Cao, X. \& Mokhtarian, P. (2005). Correlation or causality between the built environment and travel behavior? Evidence from Northern California. Transportation Research Part D, 10(6), 427-444.

Hanson, S. (1982). The determinants of daily travel-activity patterns: relative location and sociodemographic factors. Urban Geography, 3(3): 179-202.

Howell, A. \& Páez, A. Urban Geography, Household Context, and Car Ownership: A case Study in Hamilton, Ontario. Transport Research Board.

Kitamura, R., Mokhtarian, P.L. \& Daidet, L. A micro-analysis of land use and travel in five neighborhoods in the San Francisco Bay Area. Transportation, 24(2): 125-158.

La Paix, L. (2010). Relación entre la generación de viajes y la densidad urbana: caracterización de la movilidad con modelos probit ordenados. In: IX Conference of Transport Engineering (CIT), 2010.

La Paix, L. (2012). Modelling the Impact of Built Environment, Geographical Scales and Latent Constructs on Individual Travel Behaviour. Doctoral Thesis. Universidad Politécnica de Madrid.

La Paix, L., Bierlaire, M., Cherchi, E. \& Monzón, A. (2010). How urban environment affects travel behaviour? Integrated Choice and Latent Variable Model for Travel Schedules. In: Book of Selected Papers of $2^{\circ}$ International Choice Modelling Conference, vol. Stephane Hess and Andrew Daly. London, U.K.: Emerald. 
La Paix, L., Monzón, A. \& Cherchi, E. (2010). Location effects and Trip generation: evidence from Madrid Metropolitan Area. In: XII World Conference on Transport Research, 2010.

La Paix, L., Monzón, A., Cherchi, E. (2012). Modelling the relationship between urban environment and travel behaviour: policy and indicators. In: MOBIL.TUM 2012 International Scientific Conference on Mobility and Transport, vol. Munich, Germany. Munich.

La Paix Puello, L., Chowdhury, S. \& Geurs, K. Using panel data for modelling duration dynamics of outdoor leisure activities. Journal of Choice Modelling.

La Paix Puello, L. \& Geurs, K. Modelling observed and unobserved factors in cycling to railway stations: application to transit-oriented-developments in the Netherlands. European Journal of Transport Infrastructure Research, 15(1): 27-50.

La Paix Puello, L. \& Geurs, K. Integration of unobserved effects in generalised transport access costs of cycling to railway stations. European Journal of Transport and Infrastructure Research, 16(2): 385-405.

La Paix Puello, L., Olde-Kalter, M.-J., Geurs, K.T. (2017). Measurement of non-random attrition effects on mobility rates using trip diaries data. Transportation Research Part A: Policy and Practice, 106, 51-64.

McFadden, D. The measurement of urban travel demand. Journal of Public Economics, 3: 303-328.

McFadden, D. (1981). Econometric Models of Probabilistic Choice. In: Manski, C.F., McFadden, D. (eds.) Structural analysis of discrete data with economic applications, 198-272. Cambridge: MIT Press.

Monzón, A., La Paix, L., Delgado, M.A. \& Fernández, A. (2008). Influencia de la localización en los patrones de movilidad metropolitana; análisis comparado según tipologia de encuesta. Estudios de Construcción y Transportes, 108(0), 203-210. 
Paez, A., Scott, D., Potoglou, D., Kanaroglou, P. \& Newbold, K.B. Elderly mobility: Demographic and spatial analysis of trip making in the Hamilton CMA, Canada. Urban Studies, 44(1): 123-146.

Pushkarev, B. \& Zupan, J.M. (1977). Public transportation and land use policy. vol. Book, Whole. Indiana: University Pr.

Thomas, T., Puello, L.L.P. \& Geurs, K. Intrapersonal mode choice variation: evidence from a four-week smartphone-based travel survey in the Netherlands. Journal of Transport Geography. (in press).

White Mountain Survey, C. (1991). White Mountain Survey Company, City of Portsmouth Traffic/Trip Generation Study, vol. unpublished. White Mountain Survey Company, City of Portsmouth Traffic/Trip Generation Study., vol. Book, WholeOssippeee, New Hampshire.

Yáñez, M.F., Mansilla, P. \& de Dios Ortúzar, J. The Santiago Panel: Measuring the effects of implementing Transantiago. Transportation, 37(1): 125-149. 